\title{
Preliminary environmental analyses of Irish adult food consumption data to facilitate a transition to sustainable diets
}

\author{
L.B. Kirwan ${ }^{1}$, J. Walton ${ }^{2}$, A. Flynn ${ }^{3}$, A.P. Nugent ${ }^{1,4}$ and B.A. McNulty ${ }^{1}$ \\ ${ }^{1}$ School of Agriculture and Food Science, UCD Institute of Food and Health, University College Dublin, Dublin, \\ Ireland, \\ ${ }^{2}$ Department of Biological Sciences, Munster Technological University, Cork, Ireland, \\ ${ }^{3}$ School of Food and Nutritional Sciences, University College Cork, Cork, Ireland and \\ ${ }^{4}$ School of Biological Sciences, Institute for Global Food Security, Queens University Belfast, Belfast, Northern Ireland
}

The impact of the global food system is expected to exceed planetary boundaries if current dietary patterns continue. There are six key environmental limits that encompass planetary boundaries; biodiversity loss; land use change; nitrogen cycling; phosphorous cycling; water use; and climate change resulting from greenhouse gas emissions (GHGe). If these boundaries are exceeded, ecosystems and related global regulatory processes are predicted to destabilise ${ }^{(1)}$. Current estimations indicate that the safe operating space for climate change, land use change, nitrogen and phosphorous cycling have already been exceeded at a European level ${ }^{(2)}$. Hence, maintaining the impact of food systems within planetary boundaries is considered an important target in sustainable diet research. This study aims to evaluate the environmental impact of Irish diets for five environmental factors; blue water use (L), cropland use ( $\left.\mathrm{m}^{2}\right)$, greenhouse gas emissions (GHGe;kgC02eq), nitrogen use ( $\mathrm{N} ; \mathrm{kgN} / \mathrm{t})$, and phosphorous use $(\mathrm{P} ; \mathrm{kgN} / \mathrm{t})$.

Analysis was conducted using data from the National Adult Nutrition Survey (NANS; 2008-2010; 18- 90 years; www.iuna.net). Valid reported daily diets were identified using a ratio cut-off of 1.1 energy intake to basal metabolic rate $(n 3,181)^{(3)}$. GHGe and blue water use were assigned at the food code level and were sourced from UK life cycle assessment data ${ }^{(4)}$. Cropland, nitrogen, and phosphorous use were assigned at the raw primary commodity level using data from the Irish Food Conversion Model ${ }^{(5,6)}$. Planetary boundaries were sourced from published literature ${ }^{(7)}$. Multiple linear regressions and ANOVAs with Bonferroni corrections were used to evaluate environmental impact using RStudio statistical software (version 4.1.1).

The median diet had an output of $3.88 \mathrm{kgC0} \mathrm{eq}, 319 \mathrm{~L}, 13.8 \mathrm{~m}^{2}, 101 \mathrm{kgN}$ and $17.5 \mathrm{kgP}$ for females and $5.85 \mathrm{kgC} 02 \mathrm{eq}, 338 \mathrm{~L}, 16.5 \mathrm{~m}^{2}$, $118 \mathrm{gN}$ and $20.7 \mathrm{gP}$ for males per day. Higher environmental impact diets were associated with younger, male, non-smoking participants, and low energy dense diets (food kcal/g) $(P<0.05)$. When diets were grouped from low to high environmental impact, while significantly different intakes were identified for nineteen food groups by food weight, only nine of these were significant for energy intake $(P<0.05)$. The environmental impact of diets exceeded global boundaries for cropland use, GHGE, nitrogen, and phosphorous use by over $200 \%$ when assessed at the individual level, but the boundary for blue water use was not exceeded. Less than $1 \%$ of reported diets were within all planetary boundaries ( $n$ 28).

Irish diets are currently unsustainable and dietary changes are required to mitigate the climatic impact of food consumption. There is an urgent need for research at a national level to develop sustainable and healthy diets tailored to dietary preferences and cultures. Analyses on environmental impact with dietary cost, nutritional adequacy and diet modelling studies are required to ensure a successful transition to lower environmental impact diets.

\section{Acknowledgments}

The National Adult Nutrition Survey was funded by the Irish Department of Agriculture, Food and the Marine (DAFM).

\section{References}

1. Steffen W et al. (2015) Science 13, 347(6223).

2. EEA (2020) Report No. 01/2020.

3. Goldberg G.R et al. (1991) Eur J Clin Nutr 45(12), 569-81.

4. Scheelbeek P et al. (2020) BMJ Open 10(8), 037554 .

5. Springmann $\mathrm{M}$ et al. (2018) The Lancet 2(10), 451-461.

6. DAFM (2017) Report No.14/F/813

7. Chaudhary A.K et al. (2021) One Earth 2021;4(4), 531-544. 